\title{
Der Ingenieur an seinem Arbeitsplatz - gesund und kompetent!
}

Bettina Schleidt

Seit Jahren steigen psychische und psychosoziale Belastungen im Arbeitsalltag von Ingenieuren* an, was unter anderem an der zunehmenden Zahl von Erkrankungen, die beispielsweise auf permanenten Stress zurückzuführen sind, erkennbar ist. Durch die Pandemie, die seit mehr als einem Jahr Alltag und Arbeitsleben maßgeblich beeinflusst, treten diese Belastungen noch deutlicher in den Vordergrund. Nach einer kurzen Einführung werden zunächst theoretische Grundlagen dargelegt und wesentliche Begriffe definiert. Mit Blick auf den Arbeitsplatz werden potenzielle Belastungen skizziert und die Bedeutung von Kompetenzen herausgearbeitet, die unterstützen können, um mit diesen Belastungen umzugehen. Außerdem wird der Frage nachgegangen, ob Ingenieure das nötige "Kompetenz bezogene Rüstzeug" - sprich die persönlichen psychischen Ressourcen - haben, um mit den Anforderungen und Belastungen, die sich am Arbeitsplatz ergeben, adäquat umgehen zu können und welche Bedeutung der Hochschulausbildung dabei zukommt. Den Abschluss bildet ein Plädoyer für eine systematische (Neu-)Ausrichtung und regelmäßige Weiterentwicklung bzw. Anpassung der Aus- und Weiterbildung von Ingenieuren anhand von ermittelten Anforderungen bzw. Belastungen am Arbeitsplatz - nicht zuletzt basierend auf einem Constructive Alignment.

*Aufgrund der besseren Lesbarkeit wird im Artikel die männliche Sprachform gewählt. Selbstverständlich sind jeweils beide Geschlechter angesprochen.

Keywords: Belastungen, Stress, Kompetenzen, Aus- und Weiterbildung, Personalentwicklung, Faktor Mensch, Gesundheit

\section{Motivation}

Unternehmen werden durch rasch wachsende und sich verändernde Märkte, durch Trends wie Digitalisierung oder Globalisierung und aktuell durch die weltweite Pandemie vor immer neue Herausforderungen gestellt. Dabei sind vor allem die Mitarbeiter gefordert, sich immer wieder flexibel an neue Begebenheiten anzupassen und mit den psychischen Folgen, die sich daraus ergeben können, umzugehen. Daneben sorgen beispielsweise Zeit- und Termindruck oder Arbeitsverdichtung im immer stärkeren Maß für psychische und physische Belastungen. Auch das Arbeitsumfeld und die Arbeitsbedingungen - aktuell vielfach aus dem Home Office heraus - können einen ent- 
scheidenden Einfluss darauf haben, dass der menschliche Organismus ab einem bestimmten Zeitpunkt dauerhaft „unter Strom“ sprich Adrenalin steht und sich damit in einem permanenten Fight or Flight Modus befindet. Dieser Zustand mündet nicht selten in Suchtverhalten oder körperliche Erkrankungen und führt zu krankheitsbedingten Fehlzeiten oder Ausfällen der Arbeitnehmer.

In diesem Zusammenhang stellen sich folgende Fragen:

- Welche Belastungen und Beanspruchungen gibt es am typischen Arbeitsplatz eines Ingenieurs? Und welche davon könnten ggf. wie reduziert oder ganz vermieden werden? Wie könnten diese Belastungen systematisch erfasst werden?

- Wie kann der Faktor Mensch an seinem Arbeitsplatz unterstützen werden, mit den skizzierten Herausforderungen umgehen, um so langfristigen Stress zu vermeiden, sich wohl zu fühlen und dauerhaft gesund zu bleiben?

- Welche Kompetenzen (vor allem sozial und persönlich) sind aktuell und zukünftig erforderlich, um mit den Anforderungen und potenziellen Belastungen am Arbeitsplatz zurechtzukommen?

- Welche Bedeutung haben die Hochschulen bei der Vermittlung dieser Kompetenzen?

Bevor diesen Fragen nachgegangen wird, werden zunächst grundlegende Definitionen und einige ausgewählte theoretische Grundlagen vorgestellt.

\section{Definitionen}

Wie bereits erwähnt steht der gesunde und leistungsfähige Mensch (-> Faktor Mensch) bzw. Ingenieur an seinem Arbeitsplatz im Mittelpunkt dieses Beitrags. Unter Faktor Mensch wird das menschliche Wahrnehmen, Denken, Fühlen und Handeln in Abhängigkeit von der an einem Arbeitsplatz zur Verfügung stehenden Technologie und Technik, von organisationalen Einflussfaktoren wie z.B. Prozessen oder Unternehmenskultur, von sozialen Einflüssen wie der Interaktion und Kommunikation mit Kollegen und allgemeinen Umweltfaktoren verstanden (Schleidt, 2018). Abbildung 1 verdeutlicht dieses Zusammenspiel.

Alle Bereiche, mit denen der Mensch an seinem Arbeitsplatz in Interaktion steht, können sowohl eine potenzielle Belastung als auch eine Ressource sein. Unter Belastungen bzw. Belastungsfaktoren können Reize (auch im Sinne von Stressoren) verstanden werden, die vom Individuum als potenziell belastend eingestuft werden. Wirken sich diese 
Belastungen subjektiv (und in den meisten Fällen negativ) auf den Menschen aus, werden sie als Beanspruchungen bezeichnet. Psychische Beanspruchungen können z.B. das Gedächtnis oder die Aufmerksamkeit, physische Beanspruchungen z.B. das HerzKreislauf-System oder die Muskulatur betreffen (vgl. Lasogga \& Gasch, 2011).

Stress resultiert aus einer subjektiv als unangenehm empfundenen Situation, von der eine Person negativ beeinflusst wird (= Distress), im Gegensatz zum anregenden positiven Stress (= Eustress). In der transaktionalen Perspektive auf Stress steht die Inkongruenz zwischen den Anforderungen der Umwelt und den Ressourcen des Individuums im Vordergrund. Zur Bewältigung sind adäquate Coping-Strategien erforderlich (Dorsch, 2021).

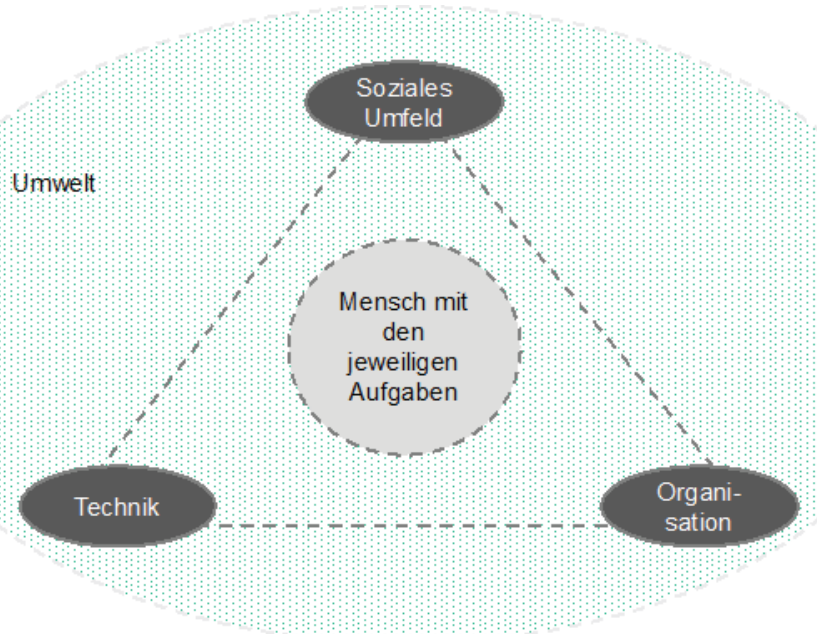

Abbildung 1: Faktor Mensch am Arbeitsplatz

Um Belastungen und Beanspruchungen zu bewältigen, kann der Mensch Ressourcen einsetzen, die ihm zur Verfügung stehen. Ressourcen sind persönliche bzw. individuelle sowie situative Potenziale, die einen direkten Effekt auf das psychische und physische Befinden haben können, und dabei helfen, beispielsweise mit Stressoren besser umzugehen (siehe Dorsch, 2021).

Als Kompetenz wird die individuelle Disposition (Aufstellung oder Anordnung) einer Person bezeichnet, ein spezifisches auf ein spezifisches Arbeitsumfeld bezogenes Verhalten zeigen zu können. Sie hat zwei Ebenen: eine nicht beobachtbare (im Sinne eines 
handlungsregulatorischen und steuernden Potenzials einer Person) und eine beobachtbare (im Sinne beobachtbarer Handlungen). Es können fachliche, methodische, soziale und personale bzw. persönliche Kompetenzen unterschieden werden. (Schleidt 2009)

Gesundheit wird analog des Verständnisses der Weltgesundheitsorganisation WHO nicht nur als das Fehlen von Krankheit sondern vielmehr als geistiges und seelisches sowie körperliches Wohlbefinden definiert.

\section{Theoretische Grundlagen}

Es gibt unterschiedliche Perspektiven, die eingenommen werden können, um die Gesundheit und das Wohlbefinden eines Ingenieurs an seinem Arbeitsplatz zu fördern. Im Folgenden werden verschiedene Ansätze dazu vorgestellt.

1. Folgt man dem salutogenetische Ansatz, werden nicht nur krankmachende (pathogene) Belastungen und Risikofaktoren sondern auch positive, gesundheitsstärkende (salutogene) Aspekte bzw. Ressourcen betrachtet. Salutogenese bezeichnet eine Konzeption, die das Entstehen und Aufrechterhalten von Gesundheit beschreibt. Stressoren sind in diesem Modell nicht per se die Ursache für Krankheit sondern Reize, die einen Spannungszustand im Individuum hervorrufen. Entscheidend ist, wie in der Folge mit diesen Reizen umgegangen wird bzw. wie sie bewältigt werden (Antonovsky, 1979).

2. Handlungstheoretische Betrachtungen stellen Gesundheit als persönliche Kompetenz bzw. Fähigkeit dar, die dazu führt, dass u.a. langfristige Ziele gebildet und verfolgt werden oder dass stabil flexibel mit sich verändernden Umweltbedingungen umgegangen wird (siehe ausführlicher Ducki \& Greiner, 1992).

Aus beiden Sichten heraus entstand das Modell in Abbildung 2, in dem der Faktor Mensch an seinem Arbeitsplatz mit den jeweiligen Aufgaben im Mittelpunkt steht. Belastungen können - wie in Abbildung 1 ersichtlich ist - in den Bereichen Technologie/Technik, Organisation, soziales Umfeld und Umwelt entstehen. Der Mensch kann diese Belastungen (sofern vorhanden) mit entsprechenden Ressourcen bewältigen und damit das Ergebnis (den Output) also seine Gesundheit, sein Wohlbefinden, sein Verhalten in einer Situation beeinflussen. Als ein wichtiger Teil dieser Ressourcen können persönliche Kompetenzen gelten, die in der Aus- und Weiterbildung erworben werden können. 


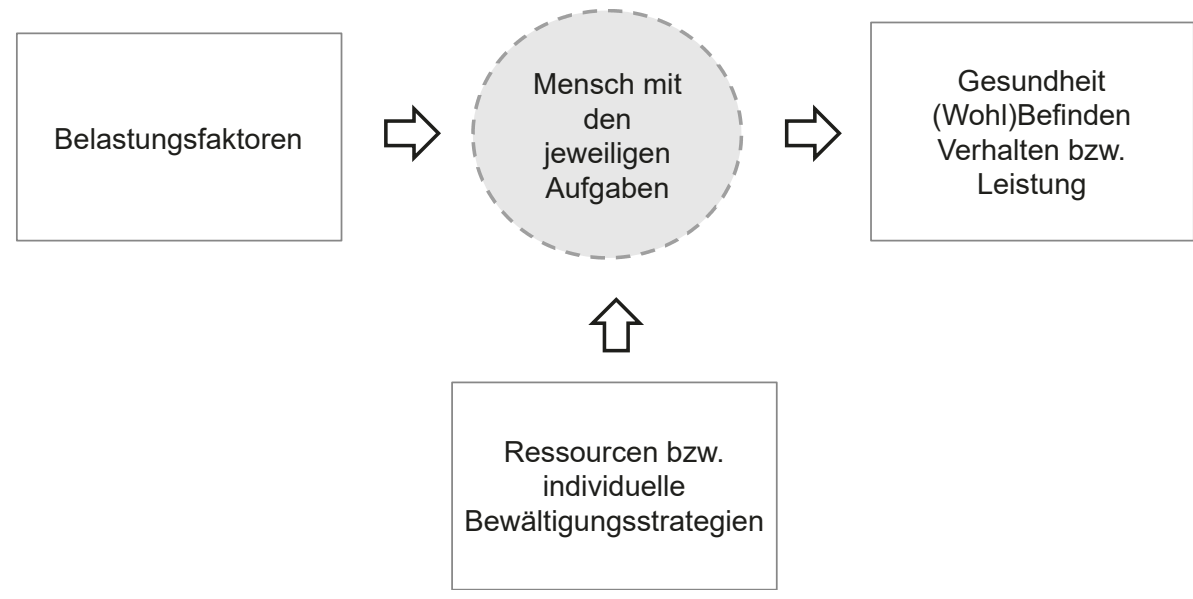

Abbildung 2: Zusammenhang zwischen Belastungen, Ressourcen bzw. individuellen Bewältigungsstrategien und Gesundheit, persönlichem Wohlbefinden und Verhalten

In einem weiteren Ansatz steht explizit die Passung zwischen Person (-> Kompetenzen) und Arbeitsplatz bzw. Tätigkeit (-> Anforderungen) im Vordergrund. Wenn ein sogenannter Fit zwischen den an einem Arbeitsplatz vorhandenen Anforderungen und den Kompetenzen, die eine Person hat, um mit diesen Anforderungen umzugehen, besteht, kann sich diese Passung in kompetentem Verhalten äußern. Ist diese Passung nicht oder nur unzureichend vorhanden, wird das Verhalten vermutlich weniger kompetent ausfallen. (Schleidt, 2009)

\section{Anforderungen und Ressourcen am Arbeitsplatz eines Ingenieurs}

In diesem Abschnitt wird das Modell aus dem Abschnitt oben auf Arbeitsplätze im Ingenieurswesen übertragen. Grundsätzlich ist die Arbeit wie bereits ausgeführt von einer zunehmenden Geschwindigkeit und Dynamik gekennzeichnet, die in vielen Fällen auch ohne bewusstes Entscheiden und Handeln eine Eigendynamik entwickelt bzw. entwickeln kann. In vielen Projekten gibt es ein wenig transparentes und nur teilweise kontrollierbares Zusammenspiel von agierenden Personen, Systemen, Daten und Prozessen und mitunter ein Über- oder Unterangebot an Informationen. Auch Faktoren wie der zeitliche Druck, der durch immer kürzer werdende Produktlebenszyklen entsteht, oder die zunehmende Komplexität der Produkte können belastend wirken. Hinzukommen können intrapsychische Belastungen, wie Ängste vor Kontrollverlust oder vor Fehlentscheidungen und damit verbundenen Konsequenzen. 
Durch den Einsatz von modernen Informations- und Kommunikationsmedien am Arbeitsplatz wird einerseits die ortsunabhängige Zusammenarbeit über Unternehmensgrenzen, Fachdisziplinen und Kulturen hinweg unterstützt, andererseits kann die wahrgenommene Komplexität durch unterschiedliche und nicht aufeinander abgestimmte Systeme steigen. Belastungen können aus der Zuverlässigkeit und Bedienbarkeit der eingesetzten Technik genauso wie aus dem Grad der Automatisierung oder der persönlichen Vertrautheit (im Sinne einer proficiency) mit der zur Verfügung stehenden Technik entstehen.

Auf der organisatorischen Ebene können fehlende Standards bei Prozessen, intransparente Arbeitsabläufe oder fehlende Verantwortlichkeiten und nicht vorhandene Absprachen für Belastungen sorgen. Im sozialen Bereich können Kommunikation und Interaktion mit Kunden, Kollegen oder Vorgesetzen belastend wirken.

Auf die Umwelt bezogene Belastungen können beispielsweise Temperatur oder Lärm am Arbeitsplatz sein.

Um diese Belastungen bewältigen zu können, bedarf es verschiedener persönlicher Faktoren wie unter anderem eine adäquate Einstellung. Diese sollte davon gekennzeichnet sein, schwierigen und belastenden Situationen etwas möglichst Positives abzugewinnen. Probleme werden als Herausforderungen betrachtet, die der eigenen Entwicklung dienen und den Erfahrungshorizont erweitern (-> Positive Psychologie). In der Psychologie wird in diesem Zusammenhang immer häufiger von der Resilienz als der persönlichen Widerstandskraft im Umgang mit Krisen und herausfordernden Situationen gesprochen.

Neben weiteren Faktoren wie Erfahrungen oder Wissen sind persönliche Kompetenzen eine hilfreiche Ressource zur Bewältigung der Anforderungen am Arbeitsplatz. Dabei treten fachliche und methodische Kompetenzen immer weiter in den Hintergrund. Stattdessen kommt es immer stärker auf Kompetenzen wie Selbstführung, sich bewußt entspannen zu können, Konzentrationsfähigkeit und Fokus auf das Wesentliche, Situationsbewusstsein oder die Fähigkeit, unter Unsicherheit Entscheidungen treffen zu können, an.

\section{Kompetenzorientierung in Aus- und Weiterbildung}

In diesem Abschnitt wird der Frage nachgegangen, welchen Beitrag eine kompetenzorientierte Aus- und Weiterbildung leisten kann, damit Ingenieure an ihrem Arbeitsplatz mit Anforderungen und Belastungen angemessen umgehen können und gleichzeitig persönliche Gesundheit und Wohlbefinden gefördert werden. 


\section{Hochschulausbildung}

Aus den Anforderungen, die Arbeitgeber an Berufsanfänger stellen, ergeben sich deutliche Hinweise auf die Ausrichtung der universitären Ausbildung. Bei der IREG-7 Konferenz der sogenannten International Ranking Expert Group (IREG), die 2014 in London stattfand, wurden unter anderem folgende weichen bzw. überfachlichen Kompetenzen genannt, die der ideale Bewerber nach Abschluss seiner Ausbildung mitbringen sollte:

- Fähigkeit, mit Menschen, die unterschiedliche berufliche Hintergründe besitzen und aus verschiedenen Ländern stammen, kooperativ zusammen arbeiten zu können

— Fähigkeit, neue Kompetenzen und Verhaltensweisen in Abhängigkeit von sich verändernden Rollenanforderungen zu entwickeln

- Persönliche Flexibilität, sich zusammen mit einem schnell entwickelnden Markt und einer globalen Wirtschaft zu verändern

— Fähigkeiten, komplexe Informationen schnell aufzunehmen

- Hohes Maß an Selbstbewusstsein (-> self awareness) und Selbst-Management

- Interdisziplinäres Wissen, z. B. Sprachen, technische Fähigkeiten (ICEF Monitor, 2014)

Ziel jeder Hochschulausbildung ist, die Studierenden bestmöglich auf Anforderungen im Berufsleben vorzubereiten und ihnen das Wissen und die Kompetenzen zu vermitteln, die am zukünftigen Arbeitsplatz benötigt werden, um mit Anforderungen und Belastungen gelassen umzugehen. Die Hochschulrektorenkonferenz betont die kompetenzorientierte Gestaltung von Studium und Lehre, in dem hervorgehoben wird, dass Studierende im Studium neben fachlich-wissenschaftlichen Kompetenzen auch Fähigkeiten entwickeln, die sie in praktischen Einsatzfeldern anwenden, anpassen, reflektieren und weiterentwickeln können. (Hochschulrektorenkonferenz 2012).

Diese kompetenzorientierte Gestaltung sollte unter anderem von einem neuen Rollenverständnis der Lehrenden als Berater und Begleiter individueller Lernprozesse und einem neuen Rollenverständnis der Studierenden, gekennzeichnet von aktivem und (weitgehend) selbst organisiertem Lernen, flankiert sein. Auch kompetenzorientierte Prüfungsformen oder begleitende Evaluation und Qualitätssicherung sind wichtige Elemente. 


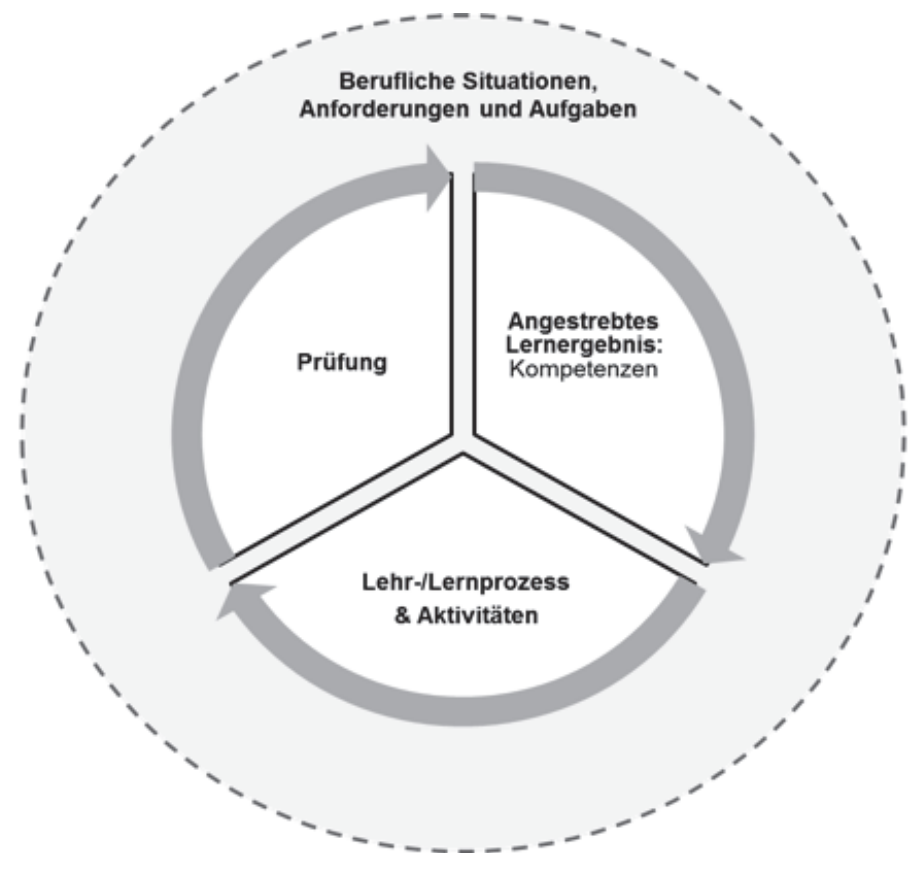

Abbildung 3: Constructive Alignment in der Hochschullehre (Schleidt, 2016)

In diesem Zusammenhang spielt das sogenannte Constructive Alignment eine wichtige Rolle. Abbildung 3 zeigt, wie bei der Entwicklung von Curricula ausgehend von der nach der Ausbildung angestrebten beruflichen Tätigkeit und den damit verbundenen Anforderungen das angestrebte Lernergebnis und Lernziel (-> Kompetenzen), die passende Lehr-/Lernsituation und schließlich die Prüfungsform in Einklang miteinander gebracht werden können. (Schleidt, 2016)

Damit ist die Herausforderung verbunden, dass Lehrende an Hochschulen auf dem Laufenden sind, welche Anforderungen an Hochschulabsolventen an ihren Arbeitsplätzen gestellt werden und wie sich diese über die Zeit hinweg verändern. Dieser Abgleich findet derzeit nur unzureichend und vor allem nicht systematisch statt.

\section{Fort- und Weiterbildung}

Auch in der betrieblichen Personalentwicklung gibt es verschiedene Wege, um die Kompetenzentwicklung von Mitarbeitern zu fördern. Ein Konzept ist der aus der Luftfahrt stammende Ansatz des Managements persönlicher Ressourcen. Ziel ist dabei zum einen, Mitarbeitern bewusst zu machen, welche Ressourcen ihnen an ihrem Arbeits- 
platz zur Verfügung stehen und wie sie diese zielorientiert einsetzen können. Zum anderen können Kompetenzen, die nicht oder wenig vorhanden waren, (weiter)entwickelt werden.

Als Methoden stehen neben den klassischen Trainings und Seminaren beispielsweise Coachings oder Kollegiale Beratungsangebote zur Verfügung.

\section{Fazit und Ausblick}

Um mit den skizzierten Anforderungen und Belastungen am Arbeitsplatz umgehen zu können, bedarf es stärker als bisher einer Hochschulausbildung, die sich daran orientiert, welche Kompetenzen ein zukünftiger Ingenieur benötigt, um die an ihn gestellten Anforderungen zu bewältigen und dabei gesund und leistungsfähig zu sein und zu bleiben. Neben fachlichen und methodischen Kompetenzen treten dabei im immer stärkeren Maß personale bzw. persönliche und soziale Kompetenzen in den Vordergrund. Sie gilt es in der Lehre der Zukunft stärker zu integrieren als bisher.

Um dieses Ziel zu erreichen, sollte es einen regelmäßigen und systematischen Abgleich zwischen psychischen und physischen Anforderungen bzw. Belastungen an Arbeitsplätzen und erforderlichen Kompetenzen geben. Die Hochschulausbildung sollte dann die so ermittelten Kompetenzen basierend auf einem Constructive Alignment in ihre Lehre integrieren.

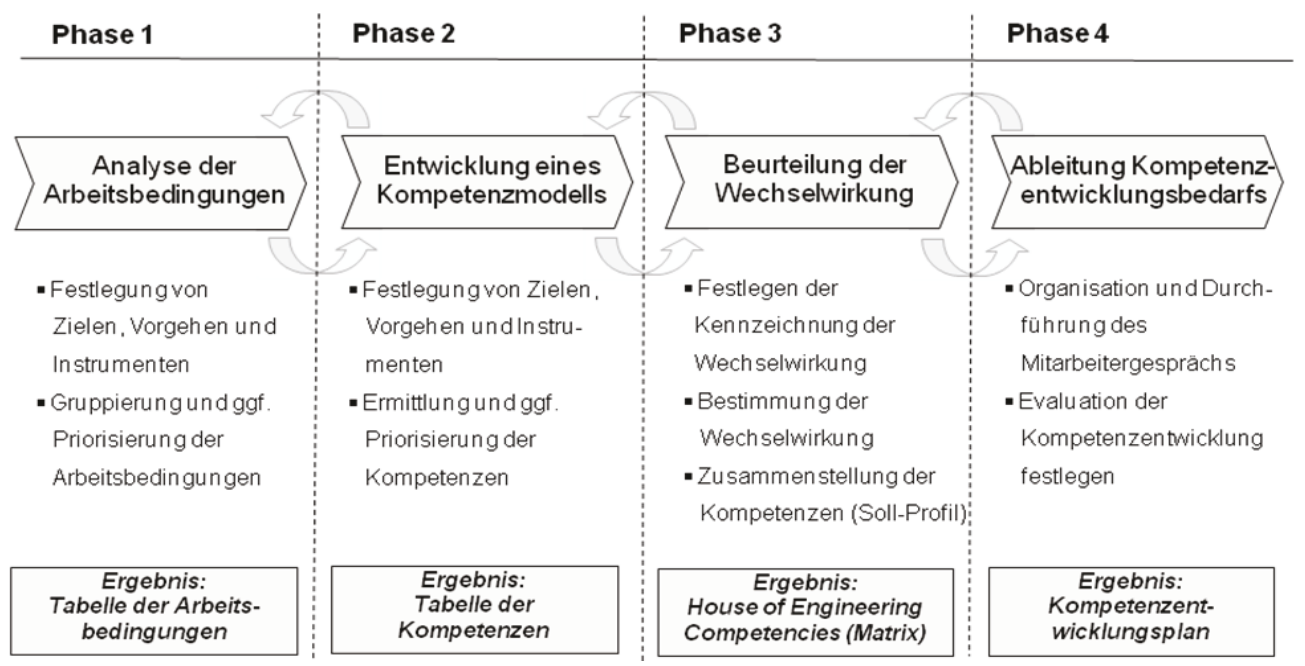

Abbildung 4: Prozess zum Abgleich von Arbeitsbedingungen und benötigten Kompetenzen (Schleidt, 2009) 
Eine Möglichkeit zur konkreten Umsetzung dieses Abgleiches könnte sein, dass parallel zu einem Forschungsprojekt ein Gremium ins Leben gerufen wird, das sich aus Experten der Industrie, Hochschullehrenden und weiteren Fachleuten zusammensetzt, und das in regelmäßigen Abständen nach einer vorgegebenen Systematik ein Kompetenz bezogenes Alignment von den Anforderungen der aktuellen und zukünftigen beruflichen Tätigkeit und den Kompetenzen, die für den Umgang damit erforderlich sind, vornimmt und daraus abgeleitet inhaltliche Anregungen oder sogar konkrete Arbeitshilfen zur Verfügung stellen könnte.

Abbildung 4 zeigt eine mögliche Vorgehensweise, die auf die beschriebene Zielsetzung übertragen werden könnte. Dabei wird aufgezeigt, wie in einem Unternehmen auf einen Arbeitsplatz bezogen ein Kompetenzentwicklungsbedarf ermittelt werden kann, der sich an bestehenden Arbeitsbedingungen orientiert. In der Phase der Beurteilung der Wechselwirkung werden die jeweiligen Arbeitsbedingungen den Kompetenzen, die zu ihrer Bewältigung erforderlich sind, gegenübergestellt und miteinander abgeglichen. Das Ergebnis ist die Ableitung des jeweiligen Kompetenzentwicklungsbedarfes, der in einer konkreten Planung zur Kompetenzentwicklung mündet.

Dieser Ablauf könnte auf die beschriebene Thematik übertragen werden und in die Konzeption einer kompetenzorientierten Lehre an Hochschulen münden.

\section{Literaturverzeichnis}

Antonovsky, A. (1979). Health, stress and coping: New perspectives on mental and physical well-being. San Francisco: Jossey-Bass.

Ducki, A., \& Greiner, B. (1992). Gesundheit als Entwicklung von Handlungsfähigkeit - Ein arbeitspsychologischer Baustein zu einem allgemeinen Gesundheitsmodell. In: Zeitschrift für Arbeits-und Organisationspsychologie, 36, 184-189.

Dorsch (2021). Lexikon der Psychologie. M.A. Wirtz (Hrsg.). Stichwortsuche. https://dorsch.hogrefe.com/, abgerufen am 12.04.2021.

Hochschulrektorenkonferenz (Hrsg.) (2012). Projekt nexus - Konzepte und gute Praxis für Studium und Lehre (2012). nexusimpulse für die Praxis Nr. 1: Kompetenzorientierung im Studium. Vom Konzept zur Umsetzung. https://www.hrk-nexus.de/fileadmin/redaktion/hrk-nexus/07-Downloads/07-02-Publikationen/impulse_Onlineversion.pdf, abgerufen am 04.03.2021.

ICEF Monitor (2014). Employability and university rankings: London conference reveals what employers want from schools. http://monitor.icef.com /2014/05/ employability-and-university-rankings-london-conference-reveals-what-employerswant-from-schools/, abgerufen am 11.04.2021.

Lasogga, F., Gasch, B. (2011). Notfallpsychologie (2. Aufl.). Heidelberg: Springer.

Schleidt, B. (2018). Psychologie sicheren Handelns bei der Arbeit. In: Brinkmann, R. (Hrsg.): Angewandte Wirtschaftspsychologie, 417-447, Halbergmoos: Pearson. 
Schleidt, B. (2016). Erfolgsfaktoren guter Hochschullehre. In: Brinkmann, R. (Hrsg.): Problembasiertes Lernen in der Ausbildung von Psychologiestudenten. Heidelberg: Heidelberger Hochschulverlag.

Schleidt, B. (2009). Kompetenzen für Ingenieure in der unternehmensübergreifenden virtuellen Produktentwicklung. Dissertation. In: Eigner, M. (Hrsg.): Schriftenreihe VPE. Band 7. Kaiserslautern: Technische Universität.

\section{Kontakt}

Prof. Dr.-Ing. Bettina Schleidt

SRH Hochschule Heidelberg

Maria-Probst-Strasse 3

69123 Heidelberg

www.hochschule-heidelberg.de 